member of the European Academy of Sciences; and a fellow of the American Physical Society, the American Association for the Advancement of Science, the
Microscopy Society of America, and the Materials Research Society. He has received the S.T. Li Prize for Outstanding Contribution in Nanoscience and
Nanotechnology, the Burton Medal from the Microscopy Society of America, and the Purdy Award from the American Ceramic Society.

\section{Mark E. Davis to give Kavli lecture on fighting cancer with nanoparticles}

$\mathbf{M}$ ark E. Davis of the California Institute of Technology has received the Fred Kavli Distinguished Lectureship in Nanoscience. At the 2011 Materials Research Society Fall Meeting in Boston, he will present his lecture, "Fighting cancer with nanoparticle medicines-

\section{MRS The nanoscale matters!" The} lecture is scheduled for Sunday, Nov. 27 at 7:00 p.m. in the Grand Ballroom of the Sheraton Hotel.

Nanoparticle medicines, or nanomedicines, have the potential to provide "game changing" ways to treat cancer. Davis will demonstrate how physical insights at the nanoscale allow for the development of nanoparticles that can function as designed to fight diseases in animals and humans. The data from humans, he said, show how two independent nanoparticle cancer therapeutics have been translated from laboratory curiosities to experimental therapeutics in human clinical trials.

Davis is the Warren and Katharine Schlinger Professor of Chemical Engineering at the California Institute of Technology and a member of the Experimental Therapeutics Program of the Comprehensive Cancer Center at the City of Hope institution. He has over 375 scientific publications, two textbooks, and over 50 patents.

His research efforts involve materials synthesis in two general areas; namely, zeolites and other solids that can be used for molecular recognition and catalysis, and polymers for the delivery of a broad range of therapeutics. He is the founder of Insert Therapeutics Inc., a company that focused on the use of cyclodextrincontaining polymers for drug delivery applications and Calando Pharmaceuticals, Inc., a company that created the first RNAi therapeutic to reach the clinic for treating cancer. He has been a member of the scientific advisory boards of Symyx and Alnylam.

Among Davis's honors are elections to the National Academy of Engineering (1997), the National Academy of Sciences (2006), and the Institute of Medicine (2011).

The Kavli Foundation supports scientific research, honors scientific achievement, and promotes public understanding of scientists and their work. Its particular focuses are astrophysics, nanoscience, and neuroscience.

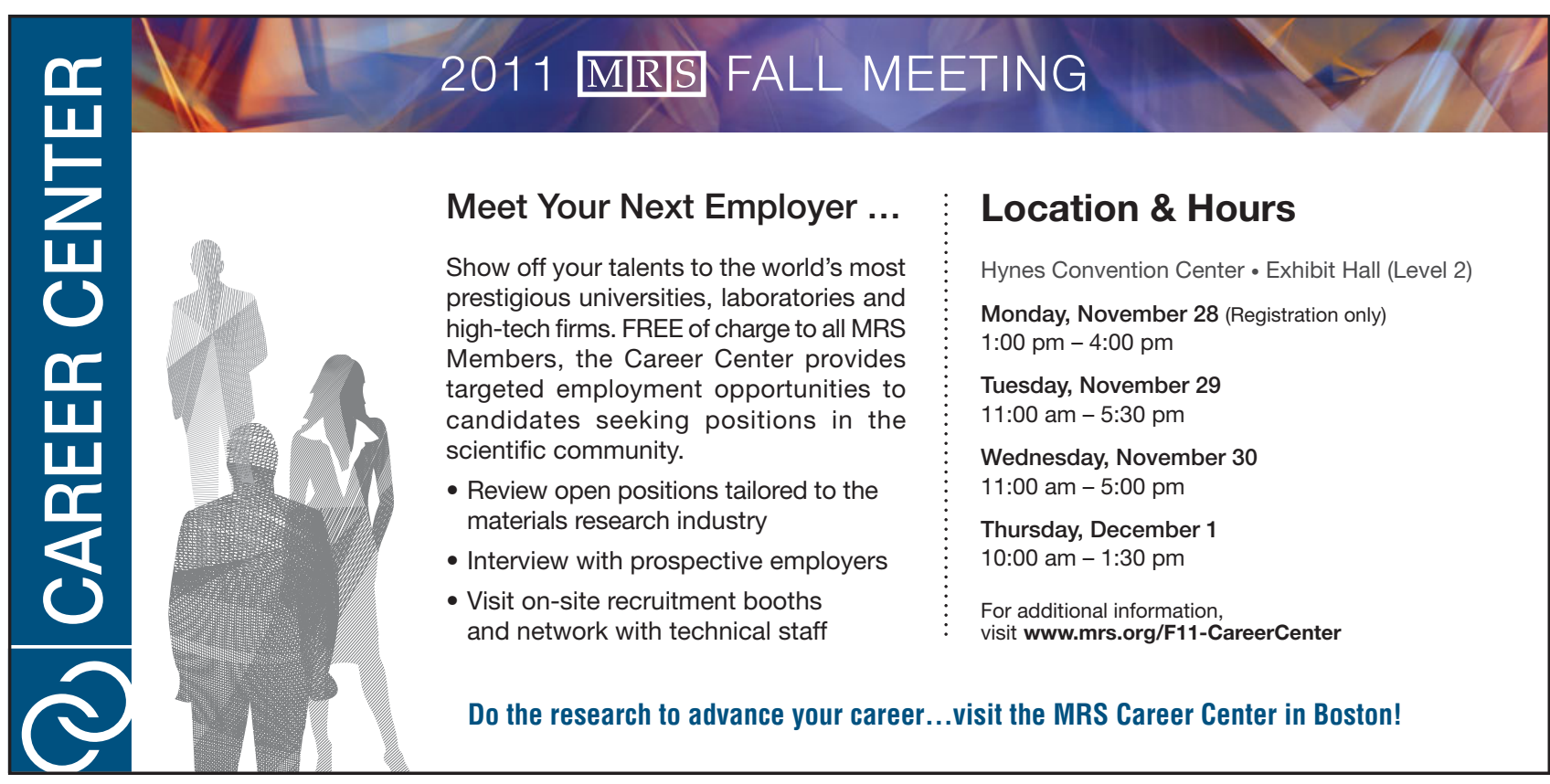



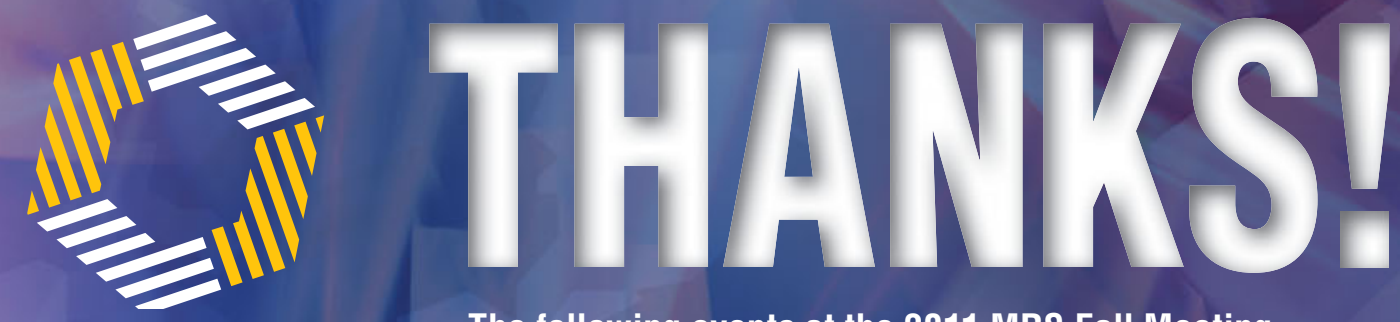

The following events at the 2011 MRS Fall Meeting have been funded, in part, by the generous contributions of these organizations.

\section{POSTER SESSIONS}

\section{ACS Publications}

MOST TRUSTED. MOST CITED. MOST READ. pubs.acs.org • Booth 125

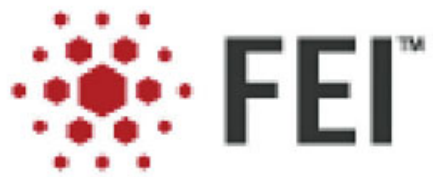

www.fei.com Booth 313

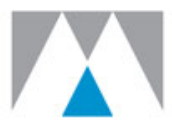

MATERIO N

www.materion.com • Booth 928

RSCPublishing

pubs.rsc.org • Booth 110

\section{COFFEE BREAKS}

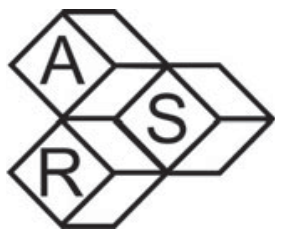

www.arscryo.com • Booth 317

WOMEN IN MATERIALS SCIENCE BREAKFAST

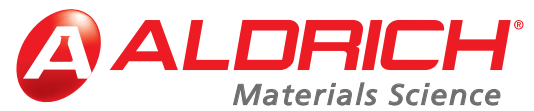

www.sigma-aldrich.com/matsci • Booth 1024

STUDENT MIXER

ACS Publications

MOST TRUSTED. MOST CITED. MOST READ.

pubs.acs.org • Booth 125

\section{BADGE LANYARDS}

\section{TED PELLA, INC.}

Microscopy Products for Science and Industry

www.tedpella.com • Booth 516 


\section{MRS FALI/MEETING}

10 Angstroms

www.10angstroms.com

Electron Beam Lithography Systems; Nanoindenters: Deposition Systems; Used Scanning Electron

Microscopes; SEM Accessories

\section{A \& N Corporation \\ Booth 1025 \\ www.ancorp.com \\ Vacuum Chambers; Vacuum Fittings; Vacuum Valves}

\section{AAAS Science \& Technology}

Booth 122

Policy Fellowships

www.fellowships.aaas.org

\section{Accelrys, Inc.}

accelrys.com

Materials Studio; Pipeline Pilot

\section{Across International LLC}

www.acrossinternational.com

Digital Drying Ovens: Lab High Temperature

Furnaces; Lab Planetary Ball Mills

\section{ACS Publications}

pubs.acs.org

Journals

\section{AdValue Technology, LLC}

www.advaluetech.com

Ceramic Labwares; Quartz Products;

Thermal Analysis Sample Pans

Advanced Diamond Technologies, Inc. Booth 718 www.thindiamond.com

NaDiaProbes (All-diamond AFM Probes);

UNCD Diamond Wafers; UNCD Electrodes

Advanced Polymer Materials Inc. Booth 1303 www.apmpolymers.com

Amphiphilic Copolymers; Functional PEGs:

Functional Copolymers

Advanced Research Systems, Inc. Booth 317 www.arscryo.com

Closed and Open Cycle Cryogenic Systems:

Cryogenic Probe Station

\section{Agilent Technologies}

www.agilent.com/find/nano

Atomic Force Microscopes; Nanoindenters;

Field Emission Scanning Electron Microscopes;

Microwave Network Analyzers

\section{AIST-NT, Inc.}

www.aist-nt.com

Atomic Force/ Scanning Probe Microscopes;

Combined AFM \& Raman Spectroscopy Systems

TERS-enabled Solutions

\section{AIXTRON SE}

Booth 424

Www.aixtron.com

MOCVD and CVD Equipment; OVPD, PVPD

and CNT Equipment; AVD and ALD Equipment

AJA International, Inc. Booth 306

www.ajaint.com

Sputtering Systems; Sputter Sources; Sputter Targets

\section{Aldrich Materials Science}

Booth 1024

www.sigma-aldrich.com/matsci

Organic Electronics; Alternative Energy

Nanomaterials
Alfa Aesar, a Johnson Matthey Company Booth 912 www alfa com

High-Purity Metals; Evaporation Materials; Ceramics

\section{Alfred University}

engineering.alfred.edu

Booth 223

Energy; Environment; Research

American Institute of Physics

Booth 117

www.aip.org

Physics Journals; Online Hosting:

Conference Proceedings

American Physical Society

publish.aps.org

Publications: Physics Journals; Online Journals

Anasys Instruments Corp.

Booth 1207

www.anasysinstruments.com

Nanoscale Infrared Spectroscopy (nanolR);

Nanoscale Thermal Analysis (nano-TA)

Andeen-Hagerling, Inc.

Booth 521

www.andeen-hagerling.com

Precision Capacitance and Loss Bridges:

Precision Reference Capacitors

Anfatec Instruments $A G$

Booth 1210

www.anfatec.de

AFM and STM; LockIn Amplifiers; SPM Controllers

Angstrom Engineering Inc.

Booth 1018

WWW.angstromengineering.com

Vacuum Deposition Systems; PVD Thin Film Systems

CVD Thin Film Systems

\section{Angstrom Sciences}

Booth 1306

www.angstromsciences.com

Magnetrons; Sputtering Material;

Sputtering Cathodes

\section{Angstrom Scientific Inc.}

Booth 807

www.angstrom.us

Electron Microscopy; Nano-manipulators

Angstrom Sun Technologies, Inc.

Booth 834

www.angstec.com

Spectroscopic Ellipsometer; Spectroscopic

Reflectometer; Microspectrophotometer;

Thin Film \& Coating Thickness Testing Tools

\section{Annealsys}

Booth 804

www.annealsys.com

RTP; RTCVD; Annealing; MOCVD; Spray-CVD; LPCVD

\section{Anton Paar USA}

Booth 906

www.anton-paar.com

Small Angle X-Ray Scattering

SAXS; X-Ray Diffraction

Applied Nanostructures, Inc.

Booth 1205

www.appnano.com

AFM Probes; MEMS; Microscope Parts

\section{Applied Surface Technologies}

Booth 1212

www co2clean com

$\mathrm{CO}_{2}$ Snow Jet Cleaning; Surface Cleaning

Substrate Cleaning

\section{Arbin Instruments}

Booth 911

www arbin com

Potentiostat/Galvanostat; Battery Test Equipment;

Electrochemical Testing

\section{Hynes Convention Center}

Level 2

Tuesday, November 29

11:00 am - 5:30 pm

Wednesday, November 30

11:00 am - 6:00 pm

Thursday, December 1

10:00 am - 1:30 pm

\section{Asylum Research}

Booth 510

www.AsylumResearch.com

Cypher AFM; MFP-3D AFM; Atomic Force/Scanning

Probe Microscopes

attocube systems AG

www.attocube.com

Booth 509

Nanopositioning Systems;

Microscopes; Interferometers

\section{Attolight AG}

Booth 821

www.attolight.com

Cathololuminescence System; SEM

AVS

www.avs.org

Publications; Membership; Training

B\&W Tek, Inc.

www.bwtek.com

Raman Spectrometers; Spectrometer Modules; Lasers

Baden-Württemberg International Booth 111

www bw-i.de

Discover Excellence-Made in Germany

Balazs NanoAnalysis

Booth 1130

www. balazs.com

Analytical Services (UPW, Chemical,

Air, Component); Thin Film/Wafer Analysis;

Material and Chamber Parts Characterization

\section{BigC: DinoLite Scopes}

Booth 828

www.bigc.com

Handheld Digital Microscope

Biolin Scientific, Inc.

www biolinscientific.com

Quartz Crystal Microbalance with Dissipation

Monitoring; Farfield Dual Polarization Interferometer;

Attension Theta Optical Tensiometer

Biologic USA

www.bio-logic.us

Electrochemical Instrumentation for Battery

and Fuel Cell Testing

Blue Wave Semiconductors, Inc.

Booth 803

www.bluewavesemi.com

Substrate Wafer Heaters; Thin Films \& Coating

Materials and R\&D Services:

PVD \& CVD Deposition Systems

Brooks Automation, Inc.

www.brooks.com

Instrumentation; Cryogenics; Robotics 
Bruker

Booth 301

www.bruker.com

X-Ray Diffraction \& Fluorescence Instruments

Raman Spectroscopy; Atomic Force Microscopy

\section{Bruker Nano Surfaces Division}

Booth $\mathbf{3 0 0}$

www.bruker.com

Atomic Force Microscopes; 3D Optical Microscopes;

Stylus Profilers; Tribometers; Nano-Micro-

and Macro-Indenters; Scratch Testers

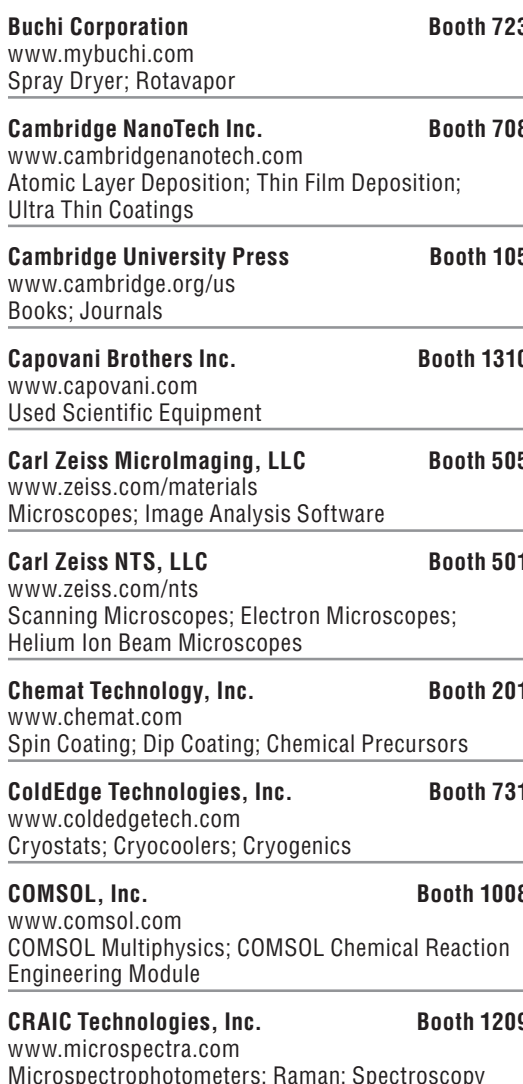

Microspectrophotometers; Raman; Spectroscopy

Cryogenic Control Systems, Inc.

Booth 733

www.cryocon.com

Temperature Controllers and Monitors; Sensors

\section{CRYSTAL GmbH}

Booth 706

www.crystal-gmbh.com

Substrate/Wafer; Laser Crystals; Optical Components

CrystalMaker Software Ltd.

Booth 929

www.crystalmaker.com

CrystalMaker; CrystalDiffract; SingleCrystal

CSM Instruments Inc.

www.csm-instruments.com

Scratch Testers; Indentation Testers; Tribometer Pin-on-Disk

\section{Cyberstar}

www.cyberstar.fr

Crystal Growth Furnaces; Crystal Growth R\&D;

Photovoltaics and Semiconductors

cyberTECHNOLOGIES USA, LLC

www.cybertechnologies.com

Optical Surface Metrology;

Optical 3D Roughness Measurement

Wafer Stress and Flatness Measurement
DCA Instruments, Inc.

Booth 520

www.dca.fi

UHV Deposition Systems; Effusion Cell;

Substrate Heater Stage

\section{DECTRIS Ltd.}

Booth 909

www.dectris.com

X-ray Detectors; Non-destructive Testing;

Single-Photon Counting

\section{Delong America Inc.}

Booth 930

www.Iv-em.com

Benchtop Combined TEM and SEM; Low Voltage TEM

\section{Denton Vacuum, LLC}

www.dentonvacuum.com

Sample Preparation Tools; High Vacuum Carbon

Evaporators; Thin Film Deposition Systems

\section{Digital Surf SARL}

www.digitalsurf.com

Surface Imaging and Analysis Software for SPMs; Microscopes; Profilometers

\section{DTF Technology GmbH}

www.dtf-technology.de

Flash Lamp Annealing Systems; Vacuum Equipment;

Thin Film Deposition

EBARA Technologies, Inc.

Booth 1120

www.ebaratech.com

Vacuum Pumps

\section{Ecopia Corp.}

Booth 1206

www.ecopia21.co.k

Variable Temperature Hall Effect Measurement

System; Probe Station; Heat Treatment System

eDAQ, Inc.

Booth 1019

www.edaq.com

Electrochemical Impedance Spectroscopy;

Electrochemistry Systems; Data Recorders

\section{Electron Microscopy Sciences/}

Booth 1010

Diatome U.S.

www.emsdiasum.com

Microscopy Supplies; Material Specimen Preparation

\section{Elsevier}

Booth 100

www.elsevier.com

Books; Journals; Electronic Products

\section{EMD Chemicals Inc.}

www.emdchemicals.com

Booth 831

\section{Energetiq Technology}

www.energetiq.com

UV Light Source; Broadband Light Source;

High Brightness Light Source

$\begin{array}{ll}\text { FEl Company } & \text { Booth } 313 \\ \text { www.fei.com } & \end{array}$

www.fei.com

Scanning Electron Microscopes;

Transmission Electron Microscopes; DualBeam

SEM/FIB Microscopes

Firefly Global

Booth 1109

www.fireflyglobal.com

Digital Video Microscopes; Wireless Digital

Microscopes; UV Microscopes

\section{First Nano}

Booth 919

A Division of CVD Equipment Corporation

www.firstnano.com

CVD Equipment; Gas/Chemical Delivery Systems Gas Abatement Systems

Fischione Instruments

Booth 309

www.fischione.com

Electron Microscope Accessories; Sample Preparation

FM Lab

Booth 1208

www.fmlab.ru

F-Mobile Energy; Energy Storage; Functional Materials
FUJIFILM Dimatix, Inc.

Booth 1007

www.dimatix.com

Materials Deposition Printer; Materials Deposition

Cartridge; Other Printheads and Systems

Gamry Instruments

Booth 1113

www.gamry.com

Potentiostats; Electrochemistry

Materials Characterization

Gatan, Inc.

Booth 600

www.gatan.com

Materials Characterization;

Nanotechnology; Photovoltaics

Geib Refining Corp.

www.geibrefining.com

Reclaim of Precious Metals; Materials, Gold

Gelest Inc.

www.gelest.com

Silanes; Silicone; Metal Organics

\section{Go!Foton}

www.gofoton.com

Fluorescence Detectors; Photonics Design;

Fiber Optics

\section{Goodfellow Corporation}

www.goodfellowusa.com

Metals and Materials for Research \& Development

Hamamatsu Corporation

www.sales.hamamatsu.com

Booth 524

Quantum Yield Measurement; NIR; VUV; Electron

Detection and Imaging

HeatWave Labs Inc.

www.cathode.com

Substrate Heaters; Cathodes; Electron Guns

\section{Herzan LLC}

www.herzan.com

Vibration Isolation; Acoustic Isolation;

Environmental Solutions

Hielscher USA, Inc

www.hielscher.com

Ultrasonic Mixers; Dispersion Technology;

Sonochemistry

Hitachi High

Technologies America, Inc.

www.hitachi-hta.com

VP/UHR Field Emission Scanning Microscopes;

Transmission Electron Microscopes; Focused Ion/ Electron Beam Systems

\section{HORIBA Scientific}

Booth 1012

Www.horiba.com/scientific

Raman; Spectroscopy; Ellipsometry

Hummingbird Scientific LLC

www.hummingbirdscientific.com

TEM Specimen Holders

Huntington Mechanica

Laboratories, Inc.

www huntvac.com

Vacuum Chambers; Vacuum Feedthroughs;

Vacuum Hardware

Hysitron, Inc.

Booth 324

www.hysitron.com

PI 95 TEM Picolndenter; PI 85 SEM Picolndenter;

TI 950 Tribolndenter

Hysitron, Inc.

Booth 417

www.hysitron.com

Nanomechanical Test Instrumentation;

TI 950 Tribolndenter; TI 750 Ubi; TS 75 TriboScope 
bss Group, Inc.

Booth 72

www.ibssgroup.com

GV10x DS Plasma Asher to reduce

hydrocarbon contamination

\section{Image Metrology A/S}

Booth 225

www.imagemet com

SPIP Image Analysis Software

\section{Imina Technologies}

www.imina.ch

Micromanipulator; Nano-prober; SEM/FIB

Accessories; In Situ Material Characterization;

Sample Preparation

$\begin{array}{ll}\text { Indigo Instruments } & \text { Booth } \mathbf{9 3 1} \\ \text { www.indigo.com } & \\ \text { Molecular Models } & \end{array}$

Molecular Models

Inel, Inc.

Booth 925

www.inel.us

Powder Diffraction; X-ray Diffraction; X-ray Detectors

\section{INFICON}

Booth 817

WW.inficon.com

Thin Film Deposition; Quartz Crystal Microbalance

Research; Vacuum Gauges and Components

Innovative Technology, Inc.

www.gloveboxes.com

Glove Boxes; Vapor Deposition Equipment; Spin

Coaters; Gas Purification Systems

Institute of International Education, Booth 221

Whitaker Program

www.whitaker.org

Intermodulation Products $A B$

Booth 1301

www.intermodulation-products.com

Atomic Force Microscopy; Surface Analysis

Multifrequency Lock-in Amplifiers

\section{International Centre}

For Diffraction Data (ICDD)

Booth 507

www.icdd.com

X-Ray Powder Diffraction; Database; Software

International Union of Crystallography Booth 118 www.iucr.org

Journals; Reference Books; Online Services

IOP Publishing

Booth 108

publishing.iop.org

Leading journals including Nanotechnology,

Science \& Technology of Advanced Materials,

Journal of Physics D: Applied Physics and MSMSE

\section{Janis Research Company, LLC Booth 408}

www janis com

Micromanipulated Probe Stations;

Cryostats; Cryocoolers

\section{Japan Science}

Booth 204

and Technology Agency

www.jst.go.jp/tt/EN/univ-ip/cips/licensing.html

Technology Licensing for Categories including New

Materials and Electronic Devices

Japan Society of Applied Physics Booth 205

www.jsap.or.jp/english

Journals, such as Applied Physics Express,

Japanese Journal of Applied Physics

JEOL USA, Inc.

Booth 601

Www.jeolusa.com

TEM; SEM; NMR

Kaufman \& Robinson, Inc.

www.ionsources.com

Ion Source; Electron Sources; Plasma Source
Keithley Instruments, Inc.

Booth 901

www.keithley.com

Sensitive Electrical Measurement Instruments;

I-V Characterization Systems

Kimball Physics, Inc.

www.kimballphysics.com

UHV Electron and Ion Guns; Cathodes; Vacuum

Chambers and Fittings

Kitco Metals Inc.

Booth 1128

pep.kitco.com

PVD Materials; Evaporation Materials;

Precious Metal Foil; Wire and Tubing

$\begin{array}{ll}\text { KLA-Tencor Corporation } & \text { Booth } 701\end{array}$

www.kla-tencor.com

Stylus Profiler; Universal Microscope

KP Technology Ltd.

www.kelvinprobe.com

Scanning Kelvin Probe; UHV Kelvin Probe;

Controlled Atmosphere Kelvin Probe

\section{Kurt J. Lesker Company}

Booth 411

www.lesker.com

Pure Targets and Materials; Vacuum Components:

Deposition Systems; Target Bonding Services

UHV Manipulation

Kyoto Environmental

Booth 219

Nanotechnology Cluster

www.kyo-nano.jp/index.php?lang=en

Mist CVD; Environmental Senso

Lake Shore Cryotronics, Inc.

www.lakeshore.com

Hall Effect Measurement Systems; Probe Stations;

Cryogenic Instruments and Sensors

Laser Quantum Inc.

www.laserquantum.com

Lasers

Leica Microsystems Inc.

Booth 316

www.leica-microsystems.com

Microscopes; Sample Preparation; Imaging Systems

Lumigntech Co. Ltd

Booth 1111

www.lumigntech.net

GaN Substrate; GaN Template; AIN Template

\section{Braun, Inc.}

Booth 421

www.mbraunusa.com

Gloveboxes \& Gas Purifiers; Vacuum Systems

Solvent Purification Systems

Mantis Deposition Ltd.

Booth 602

www mantisdeposition com

Deposition Systems; Nanoparticle Source

Sputtering Systems

Materials Preparation Center,

Booth 220

Ames Laborator

www.mpc.ameslab.gov

Rare Earths; Custom Alloys; Single Crystals

Materion Advanced Chemicals

Booth 928

www.materion com

Materials and Chemicals

\section{Vacuum Products, LLC}

www.mdcvacuum.com

High \& Ultra High Vacuum Products;

Ceramic-to-Metal Feedthroughs; UHP Weldments

Metrohm USA, Inc.

Booth 322

www.metrohmusa.com

Electrochemical Systems;

Impedance Characterizations; Sensors

\section{METTLER TOLEDO}

WwW.mt.com

MP; DSC1; TGA/DSC1; Flash DSC
Micro Materials Limited

Booth 728

micromaterials co.uk

NanoTest System; MicroTest System

Micro Photonics Inc.

Booth 1016

www microphotonics.com

X-Ray Micro CT; X-Ray Diffraction

MicroFab Technologies, Inc.

www.microfab.com

Jetlab Printing Platforms; Microdispensin

Subsystems; Vapor Generating Systems

for Sensor Calibration

Microtrac Inc.

Booth 809

www microtrac.com

Particle Size; Imaging; Surface Area

MikroMasch USA

www.spmtips.com

Silicon Probe Techniques:

Functional Imaging of Materials

MMR Technologies, Inc.

www.mmr-tech.com

Hall Effect Measurement Systems; Seebeck Effect

Measurement Systems; Variable Temperature

Microprobe Systems

Montana Instruments Corporation

Booth 1302

www.montanainstruments.com

Cryostation; Optical Cryostat

MTI Corporation

www.mtixtl.com

Muffle \& Tube Furnace; Battery Research Equipment; Single Crystal

Nano-Master, Inc.

Booth 704

www nanomaster.com

Thermal \& E-Beam Evaporators; PA-MOCVD; PECVD

Sputtering; Etching; Cleaners; Plasma Sources

NanoAnd More USA Inc.

Booth 610

www nanoandmore com

AFM Probes; Digital Optical Microscopes; Digital

Holographic Microscopes

\section{Nanofactory Instruments AB}

Booth 710

www.nanofactory.com

In situ TEM Electrical, Mechanical and Optical

Probing Systems

Nanograde LLC

www.nanograde.ch

Nanoparticles; Dispersions; Suspensions;

Functional Thin Films and Coatings:

Printable Electronic Materials

Nanolnk, Inc.

www.nanoink.net

Micro/Nano Patterning; BioMEMS; Biomimetic

Surfaces for Cell Engineering

\section{NANOLANE}

Booth 719

Www.nano-lane.com

Nanotechnology; Optical Microscopy;

Characterization Tools

NanoMagnetics Instruments Ltd.

www.nanomagnetics-inst.com

Atomic Force Microscope; Scanning Hall Probe

Microscope; Nanopositioner

NanoMaterials Innovation Center, LLC Booth 1124 www.nanomic.org

Nanomechanics, Inc.

Booth 605

www nanomechanicsinc.com

Nano-scale Mechanical Testing; Analytical Services

Software Development 
Nanonics Imaging Ltd.

Booth 1013

Www.nanonics.co.

NSOM/SNOM; AFM; AFM/Raman

Nanophoton Corporation

www.nanophoton.jp/eng/

Confocal Raman Microscope

Nanoscale Science Research Centers, Booth 1105 Department of Energy

science.energy.gov/bes/suf/user-facilities/

nanoscale-science-research-centers/

DOE's premier user centers for interdisciplinary

research at the nanoscale

Nanosurf, Inc.

Booth 707

Www.nanosurf.com

FlexAFM; LensAFM ; FluidAFM

Nanotec Electrónica S.L.

Booth 918

www.nanotec.es

Cervantes Full Mode AFM; Dulcinea SPM Controller;

WSxM Free SPM Software

\section{Nanotrons Corporation}

Booth 830

www.nanotrons.com

Functional Coatings; Nanocoating Equipment;

Nanomaterials; Nanotechnologies

Nanounity

Booth 709

www.nanounity.com

AFM; SPM; SEM; Confocal Raman; X-ray Diffraction

Nanovea

Booth 1029

www.nanovea.com

Profilometers; Nano Mechanical Testers; Tribometers

National Electrostatics Corp.

Booth 311

www.pelletron.com

MeV Accelerators: RBS/PIXE/AMS

Ion Beam Components

\section{National Nanotechnology}

Infrastructure Network

www.nnin.org

Nanofabrication; Nanotechnology

National Reconnaissance Office

dii.westfields.net

Research \& Development Funding; Remote Sensing; Innovative Research

\section{Nature Publishing Group}

www.nature.com

Medical \& Scientific Journals

\section{NBM Design, Inc.}

www.nbmdesign.com

Pulsed Laser Deposition; Custom Systems;

Deposition Technology

\section{Neaspec GmbH}

www.neaspec.com

Nanoscale Infrared Spectroscopy: Confocal Raman

Microscopy/Spectroscopy; Aperturless NSOM/SNOM

Neocera, LLC

Booth 716

www.neocera.com

Pulsed Laser Deposition Systems; Pulsed Electron

Deposition Systems

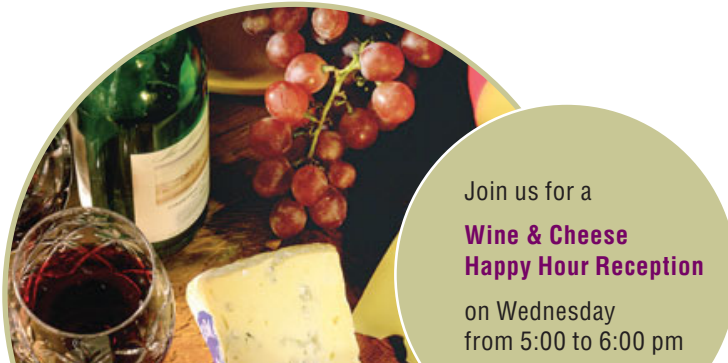

Netzsch Instruments N.A. LLC

www netzsch-thermal-analysis.com

Thermal Analysis; Thermal Conductivity; Therma Expansion; Calorimetry

\section{NIST/MSD}

Booth 216

www.nist.gov/srm

Standard Reference Materials;

Data and Calibration Services

Nor-Cal Products, Inc.

Booth 425

www.n-c.com

Vacuum Components \& Chambers;

Flanges \& Fittings; Valves

NT-MDT Co.

Www.ntmdt.com

SPM/AFM/STM; Raman TERS; Spectroscopy

\section{Oerlikon Leybold Vacuum}

www oerlikon com/leyboldvacuum

Thin Film PVD Coating Systems; Vacuum Equipment; Leak Detectors

\section{Ohio Carbon Blank Inc.}

Booth 910

www ohiocarbonblank com

EDM Graphite Electrodes; EDM Tooling

\section{Olympus America Inc.}

www olympusamerica.com

Inverted Metallographs; Upright Microscopes;

Stereo Microscopes; Filter Inspection

\section{Omicron NanoTechnology USA}

Booth 617

Omicrom Namon-instruments. com

UHV SPM; Surface Science Instrumentation; MBE

omt optische messtechnik gmbh

Booth 611

www.omt-instruments.com

TFA Reflectometer; TFA Ellipsometer;

Optical Metrology Tools

OriginLab Corporation

www.originlab.com

Origin Software

\section{Oxford Applied Research}

www.oaresearch.co.uk

Nanocluster Source; Mini e-Beam; RF Plasma Source

Oxford Instruments America Inc. Booth 621 WwW.oxford-instruments.com

Cryogenic Systems; Microanalysis; Etching,

Deposition and Growth Systems

Oxford University Press
www.oup.com/us

Publications

\section{PANalytical Inc.}

Booth 1000

www.panalytical.com

X-ray Diffraction (XRD); Computed Tomography (CT)

Small Angle X-ray Scattering (SAXS):

$\mathrm{X}$-ray Fluorescence (XRF)

\section{Park Systems Inc.}

www.parkafm.com

Atomic Force Microscopes

\section{Pascal Technologies Inc.}

www.pascaltechnologies.com

Vacuum Systems; Vacuum Equipment

Leak Detectors

PCS Instruments

www.pcs-instruments.com

Tribology Test Equipment

\section{PerkinElmer}

www.perkinelmer.com
Booth 410

Booth 1108

Booth 920

Booth 1100
Photon Technology International, Inc.

Booth 923

www.pti-nj.com

Steady State Photoluminescence; Time-Resolved Photoluminescence; NIR Photoluminescence

Physical Electronics

Booth 712

www.phi.com

Scanning Auger; SIMS; XPS; ESCA; TOF-SIMS;

Materials Analysis; Surface Analysis

Phytron, Inc.

Booth 819

www.phytron.com

Stepper Motors; Positioning Systems; Control Units

Plasma-Therm LLC

Booth 606

www plasmatherm com

Semiconductor Manufacturing Equipmen

Plasmaterials, Inc.

Booth 321

www.plasmaterials.com

Sputtering Targets; Backing Plates

Evaporation Materials

Plasmionique Inc.

www.plasmionique.com

Sputter Deposition Systems;

ICP Reactors and Sources

Postnova Analytics Inc.

www postnova.com

Field-Flow Fractionation Instruments

Powder Processing

and Technology, LLC

www pptechnology.com

Electronic Materials Process; Sustainable Energy

Materials Process; Advanced Ceramic

Materials Process

Princeton Scientific Corp.

www.princetonscientific.com

Metal Crystals; Laser Crystals; Oxide Crystallin

Materials

Process Materials, Inc.

www.processmaterials.com

Materials; Sputtering Targets; Evaporation Materials;

Cylindrical Cathodes

Protochips, Inc.

Booth 320

www.protochips.com

Microscopes, Electron Microscopy

\& Instrumentation; Nanotechnology;

Biological, Biomedical, Bio-related Sciences

PVD Products, Inc.

www.pvdproducts.com

Booth 1200

Pulsed Laser Deposition Equipment;

Sputtering; Evaporation

Quantum Design, Inc

www.qdusa.com

Booth 511

Physical Property and Magnetic Property

Measurement Systems

\section{R.D. Mathis Company}

www.rdmathis.com

Booth 1106

High Vacuum Evaporation Sources; Power Supplies; Gas Purifier

Radiant Technologies, Inc.

www.ferrodevices com

Ferroelectrics; Multiferroics; Piezoelectrics

Refining Systems, Inc.

www.refiningsystems.com

Sputtering Targets; Evaporation Materials;

Wires and Tubing

Renishaw Inc.

www.renishaw.com

Raman Microscopes; Spectrometers
Booth 422

Booth 1009 s;

Booth 623 
Research and PVD

Booth 832

Materials Corporation

www.pvdmaterials.com

PVD Materials; Sputtering Targets;

Evaporation Sources

Resodyn Acoustic Mixers, Inc

Booth 1112

www.resodynmixers.com

LabRAM; RAM5; RAM55

RHK Technology, Inc.

Booth 603

www.rhk-tech.com

SPM Universal Controls; UHV STM

UHV AFM/STM

\section{Rigaku Americas Corporation \\ www.rigaku.com \\ X-ray Diffraction Systems: \\ Small Angle X-ray Scattering Systems}

Booth 517

Rigaku Innovative Technologies, Inc. Booth 1309 www.rigaku.com

Multilayer X-ray Optics; X-ray Monochromators;

Microfocus X-ray Generators; X-ray CCD Cameras;

Rotary Motion Feedthroughs

Rocky Mountain Vacuum Tech, Inc. Booth 323

www.rmvac.com

Vacuum Systems; Vacuum Components

\begin{tabular}{ll}
\hline RSC Publishing & Booth 110 \\
Www.rsc.org/publishing &
\end{tabular}

Journals; Books

\section{SAGE Publications}

Booth 124

www.sagepub.com

Books; Journals

Scientific Computing \& Modelling NV Booth 1122 www.scm.com

Chemistry Software; Molecular Software; Density Functional Theory

\section{Seki Technotron USA}

Booth 801

www sekicydsolutions.com

Microwave Plasma CVD Systems; Hot Filament CVD

Systems; Plasma CVD Systems

Semicore Equipment Inc.

Booth 1211

www semicore com

Sputtering Systems; Evaporation Systems;

Custom PVD Systems \& Components

Shanghai Optics Inc.

Booth 922

www shanghai-optics.com

Deposition Equipment Processes \& Materials;

Materials \& Chemicals; Vacuum

Systems \& Components

Solartron Analytical (AMETEK)

Booth 917

www.solartronanalytical.com

ModuLab MTS; 1260 Impedance Analyzer;

Cryostats \& Furnaces

South Bay Technology, Inc.

www.southbaytech.com

Ion Beam Sputter Deposition; Reactive Ion Etcher;

Plasma Cleaner/Trimmer

SP Scientific
www.Spscientific.com/Companies/

SPECS Surface Nano Analysis GmbH Booth 702

www.specs.com

PHOIBOS Energy Analyzer; SPM 150 Aarhus; JT-STM;

FE-LEEM P90

\section{SPEX SamplePrep LLC}

Booth 905

www.spexsampleprep.com

Mixer/Mills; Freezer/Mills; X-Press
SPI Supplies

Booth 1202

Division of Structure Probe, Inc.

www.2spi.com

Supplies for Electron Microscopy; Plasma Etching

Systems; TEM Membrane Grid

\begin{tabular}{ll}
$\begin{array}{l}\text { Springer } \\
\text { Www.springer.com }\end{array}$ & Booth 101 \\
Books; Journals; E-Books & \\
\hline STAIB Instruments, Inc. & Booth 717 \\
WWw.staibinstruments.com & \\
RHEED; Spectrometers; Surface Analysis &
\end{tabular}

RHEED; Spectrometers; Surface Analysis

Strem Chemicals, Inc.

Booth 825

www.strem.com

Chemicals; Nanomaterials; MOCVD,

CVD and ALD Precursors

Super Conductor Materials, Inc.

Booth 618

www scm-inc com

Sputtering Targets; Evaporation Materials; Crucibles

SVT Associates, Inc.

Booth 420

www.svta.com

Molecular Beam Epitaxy; Pulsed Laser Deposition

Atomic Layer Deposition

\section{TA Instruments}

Booth 1101

www.tainstruments.com

DSC; TGA; Flash Diffusivity

\section{Taylor \& Francis-CRC Press}

Booth 104

www.taylorandfrancis com

Books; Journals; Online Products

\section{TCI America}

Booth 607

www.tciamerica.com

Fine Organic Chemicals; cGMP; Custom Synthesis

TDK-Lambda Americas Inc.

www.us.tdk-lambda.com/hp

Genesys ${ }^{\mathrm{TM}}$ Programmable AC/DC Power Supplies

\section{Ted Pella, Inc.}

www tedpella.com

Vacuum Coaters; Calibration; Microscopy Sample

Preparation \& Supplies/Accessories

Tescan USA Inc.

Booth 1104

www tescan-usa com

Electron Microscopes; Dual BeamSEM/FIB

Microscopes; Time of Flight TOF SIMS

The Mellen Company, Inc.

Booth 902

www mellencompany com

Furnaces; Crystal Growth; Custom Designs

\section{Thermionics Vacuum Products}

www thermionics com

Sample Handling; Evaporation Sources; Gate Valves

\section{Thermo Scientific}

www.thermoscientific.com/materialscienc

EDS, WDS, EBSD; Raman; XPS

Toho Technology

www.tohotechnology.com

FLX-2320-S Thin Film Stress Measurement System;

FP Series Stylus Profilers

Topcon Positioning Systems, Inc. Booth 1030 www.topconsem.com

Aquila Hybrid SEM/Optical microscope

TREK, INC.

Booth 711

www.trekinc.com

High-Voltage Amplifiers; Contacting/Noncontacting

Electrostatic Voltmeters; Surface Charge/

Resistance Measurement rion Technology, Inc.

Booth 1006

www.triontech.com

Reactive Ion Etcher (RIE); Plasma Enhanced Chemica Vapor Deposition (PECVD); Stripping Systems

TSI Inc.

Booth 921

www.tsi.com

Research Optical Particle Sizer; Water-Based

Condensation Particle Counters; Counters \& Sizers

UES, Inc.

Booth 1117

www.ues.com

RoboMet.3D; Advanced Coatings for Die Casting

ULVAC Technologies, Inc.

www.ulvac.com

Seebeck Coefficient; RTP Annealing Furnace;

Thermal Diffusivity

USHIO America, Inc.

Booth 1118

www ushio com

Electron Beam; Equipment; Custom Building

VAT, Inc.

Booth 916

Www.vatvalve.com

Vacuum Valves; Valves; Control Valves

VG Scienta, Inc.

www.vgscienta.com

Surface Analysis Systems \& Components; UHV

Systems \& Components;

Valves; Sample Manipulators

Wafer World Inc.

Booth 1020

www waferworld com

Silicon Wafers; Germanium Wafers; GaAs Wafers

Wiley

Booth 114

www.wiley.com

Books; Journals; Databases

WITec Instruments Corp.

www.witec-instruments.com

Confocal Raman Microscopy; Scanning Near-Field;

Raman AFM

J.A. Woollam Company, Inc.

www.jawoollam.com

Spectroscopic Ellipsometers; Measurement Services

World Scientific Publishing Co. Booth 1121

www.worldscientific.com

Books; Journals

XEI Scientific, Inc.

www evactron com

De-contaminators for SEMs; De-contaminators

for TEMs; Lower-Power RF Supply

$\mathrm{XOS}$

www.xos.com

Polycapillary Optics; Doubly-curved Crystal

X-Beam-X-ray Source with Optics

Yeagle Technology Inc.

www.ytionline.com

Custom Vacuum Systems;

On-site Service; Vacuum Components

Zeta Instruments

www.zeta-inst.com

3D Optical Profiler; 3D Microscope;

Film Thickness Measurements

\section{Zygo Corporation}

www.zygo.com

High Precision Measurement Systems;

Optics; Optical Design/Assembly 